\title{
Recurrent pubic osteomyelitis secondary to vulvar abscess in a post-menopausal female
}

\begin{abstract}
We present the case of a 58-year-old gravida 2, para 2 post-menopausal female who presented with a vulvar abscess which later progressed to osteomyelitis of the pubic symphysis. Osteomyelitis of pubic symphysis secondary to vulvar abscess is a rare complication, however, can be associated with significant morbidity. Although rare, most documented cases in obstetrics and gynecology occur after vaginal delivery in the postpartum period. High degree of comorbidities in affected patients, as well as the limited anatomy of the pelvic bones, makes treatment of pubic osteomyelitis challenging. Prompt diagnosis and treatment is recommended to decrease patient morbidity and mortality, and oftentimes requires prolonged periods of treatment by multidisciplinary teams.
\end{abstract}

\author{
Volume I2 Issue I - 202 I
}

\author{
Jino Park,' Kristin L Martin, ${ }^{2}$ Alexis Svokos ${ }^{2}$ \\ 'Geisinger Commonwealth School of Medicine, USA \\ 2Department of Obstetrics and Gynecology, Geisinger Medical \\ Center, USA
}

\begin{abstract}
Correspondence: Alexis Svokos, MD, Department of Obstetrics and Gynecology, Geisinger Medical Center, $100 \mathrm{~N}$ Academy Ave, Danville PA I782I, USA, Tel 570-27I-6343, Email asvokos@geisinger.edu
\end{abstract}

Received: January 12, 2021 | Published: January 22, 2021

\section{Case report}

Our patient is a 58 -year-old gravida 2, para 2 post-menopausal female who presented to the Emergency Department with a 5-day history of fever and pelvic pain. She reported documented temperature at home of $102^{\circ} \mathrm{F}$, and history of new onset pelvic pain, rated as $8 / 10$ and unrelieved by acetaminophen use at home. She reported the pain as generalized pelvic pain and radiating to her inner thighs bilaterally. The pain is exacerbated by walking and movement. Her prior medical history is significant for obesity, type 2 diabetes mellitus, chronic hypertension, osteoarthritis and depression. Her gynecologic history is significant for two prior uncomplicated term vaginal deliveries, and menopause without any debilitating symptoms. She denied any prior history of gynecologic surgery, abnormal pap smear or sexually transmitted infection. She denied any new sexual partners or recent sexual activity.

Upon evaluation, she was found to be febrile with a temperature of 38.6 Celsius, blood pressure of $123 / 70 \mathrm{mmHg}$, pulse 86 beats per minute, and respiratory rate of $18 / \mathrm{min}$. Her lab work revealed white count of $10.12 \mathrm{~K} / \mu \mathrm{L}$, hemoglobin of $10.0 \mathrm{~g} / \mathrm{dL}$ and hematocrit of $32.4 \%$, platelets of $249 \mathrm{~K} / \mu \mathrm{L}$, lactic acid of $0.9 \mathrm{mmol} / \mathrm{L}$, and CRP $209 \mathrm{mg} / \mathrm{L}$. Gonorrhea, chlamydia and urine cultures were negative. Blood cultures were obtained.

On physical exam, no evidence of infection was noted. Sterile speculum exam and pelvic exam were completed with findings of normal physiologic discharge and tenderness to palpation of the anterior and posterior vaginal walls. Transvaginal pelvic ultrasound and computed tomography (CT) of the abdomen and pelvis were obtained and found to be unremarkable. MRI of the spine was negative for osteomyelitis, epidural or paraspinal abscess.

Based on patient's overall clinical picture, there was concern for pelvic inflammatory disease and patient was initiated on cefoxitin $2 \mathrm{~g}$ IV every six hours and doxycycline $100 \mathrm{mg}$ orally every 12 hours. On hospital day two, patient remained febrile and her blood cultures revealed MRSA bacteremia. Infectious disease (ID) consult was obtained and per ID recommendations patient was initiated on IV vancomycin 1,500mg IV every 12 hours, with a target trough of 15-20. Patient underwent transthoracic echo which revealed no vegetations or evidence of infection. On hospital days three through seven, patient was continued on IV antibiotic therapy. She remained febrile; however, fever curve was improving. Patient reported significant improvement of her symptoms.

On hospital day eight, she developed right labial edema, and repeat assessment by the gynecologic team was performed. Physical exam of the vulva showed no evidence of erythema, induration or fluctuance concerning for an abscess and no palpable area to drain. On hospital day nine, patient was found to be afebrile, and repeat blood cultures were obtained and remained positive for MRSA. Intravenous vancomycin treatment was discontinued, and patient was initiated on IV daptomycin $1 \mathrm{~g}$ daily. She continued to be afebrile during remaining hospital stay, with negative repeat blood cultures on hospital day 12. Patient's pelvic pain improved, and she did not experience any worsening vulvar edema. She was discharged home on hospital day 17 with plans for ongoing outpatient IV daptomycin treatment for four weeks.

The patient presented to gynecology clinic for outpatient follow up three weeks after hospital discharge. She reported no further febrile episodes, however, her pelvic pain had returned. On repeat pelvic exam, a $6 \mathrm{~cm}$ firm, well circumscribed, non-fluctuant, tender mass was palpated on right anterior vulva. Repeat $\mathrm{CT}$ pelvis was obtained and was remarkable for a $5.0 \times 2.7 \times 6.8-\mathrm{cm}$ rim enhancing right labial abscess with concern for the superior aspect of the abscess extending to the pubic symphysis (Figure 1) (Figure 2).

Patient was then admitted to the hospital and orthopedics as well as infectious disease specialists were consulted. She underwent incision and drainage as well as surgical debridement of right vulvar abscess. At the time of her surgical intervention, orthopedics team was available, and per their exam and evaluation, it was deemed there was no involvement of the pubic symphysis and no concern for pubic osteomyelitis. Wound cultures were obtained intra-operatively, and post-operatively, patient was maintained on intravenous vancomycin, with a planned duration of treatment for 6 weeks post-operatively. Daily wound care was provided with wet to dry dressing changes. Patient was discharged home on post-operative day 4, on daily IV Vancomycin and daily home health visits would wound packing. 


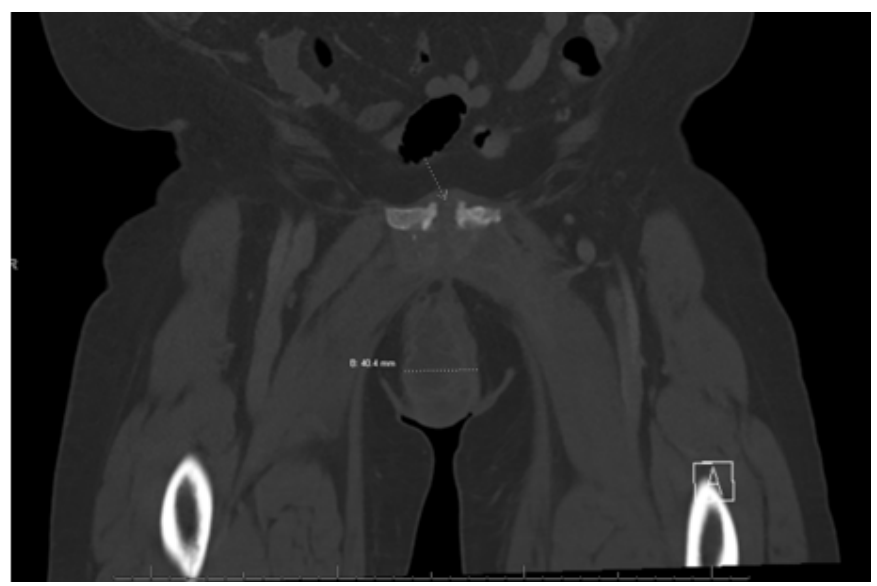

Figure I Coronal view of CT pelvis showing rim enhancing right labial abscess with concern for the superior aspect of the abscess extending to the pubic symphysis.

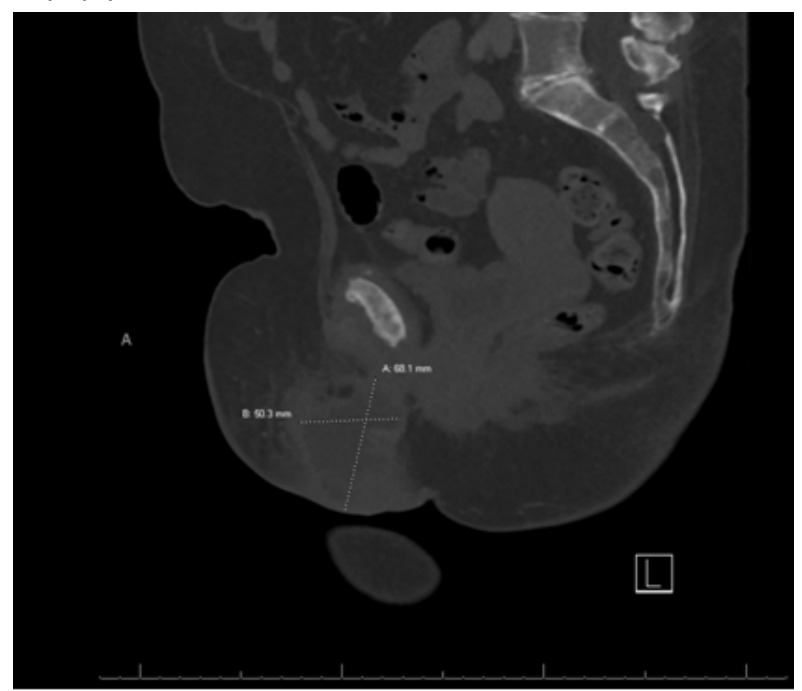

Figure 2 Coronal view of CT pelvis showing rim enhancing right labial abscess with concern for the superior aspect of the abscess extending to the pubic symphysis.

She had close outpatient follow-up with weekly visits in the ambulatory GYN clinic. On post-operative day 63, the patient presented to clinic stating that she saw "hard, black, plastic like material" protruding from her vulvar wound. Upon further evaluation and review of operative records, there was no indication for retained foreign material. Therefore, the decision was made to have the material evaluated by pathology for further identification. Pathologic evaluation of material revealed the object to be fragment of necrotic bone with adjacent neutrophils. At this time, patient was re-admitted to the hospital due to poor wound healing and concern for pubic osteomyelitis.

MRI of the pelvis was obtained and showed interval resolution of right vulvar abscess and persistent sinus tract extending from the skin at the level of the prior vulvar abscess extending into the pubic symphysis (Figure 3). Orthopedic surgery and infectious disease specialties were once again consulted for further recommendations and management. Per infectious disease recommendations, repeat blood and wound cultures were obtained. She then underwent additional surgical management by gynecology and orthopedics. Gynecology debrided the right vulvar skin, subcutaneous tissue and wound to the level of the pubic symphysis (Figure 4). Additionally, through an abdominal approach, orthopedic team $\mathrm{p}$ obtained access via a Pfannenstiel incision to evaluate the pubic symphysis and surrounding bone (Figure 5). The infected bone and surrounding tissue structures were thoroughly debrided and copiously irrigated, and wound was packed with $1 \mathrm{~g}$ of vancomycin powder. Wound cultures obtained intra-operatively showed anti-microbial resistance to vancomycin, therefore per infectious disease recommendations, she was transitioned to daptomycin $10 \mathrm{mg} / \mathrm{kg}$ every $24 \mathrm{hrs}$ and ceftaroline $600 \mathrm{mg}$ IV every $8 \mathrm{hrs}$. Ongoing wound care was provided with daily wet to dry dressing changes. Patient was discharged home in stable condition on post-operative day 6 and had daily home health visits for administration of IV antibiotics and wound care. She continued to be seen weekly in the ambulatory gynecology clinic. Her vulvar wound as well as abdominal incision showed progressively marked improvement and healing.

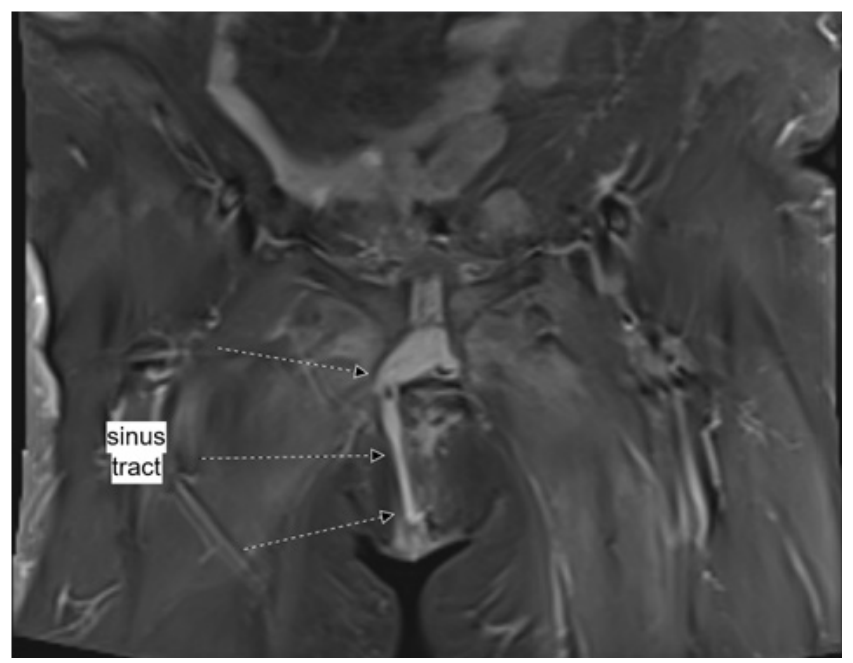

Figure 3 Coronal view of pelvic MRI showing sinus tract extending from skin of right vulvar abscess to the symphysis pubis.

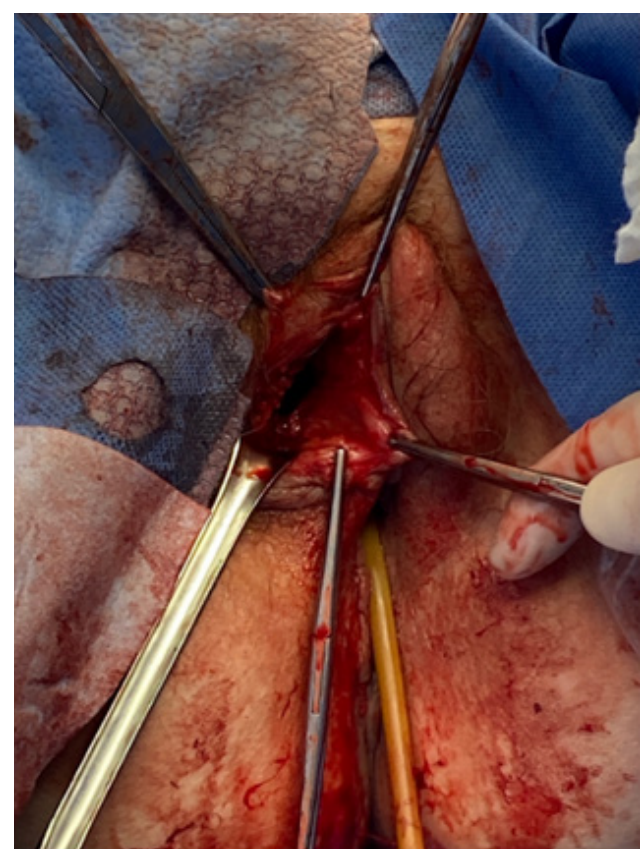

Figure 4 Gynecology debrided the right vulvar skin, subcutaneous tissue and wound to the level of the pubic symphysis. 


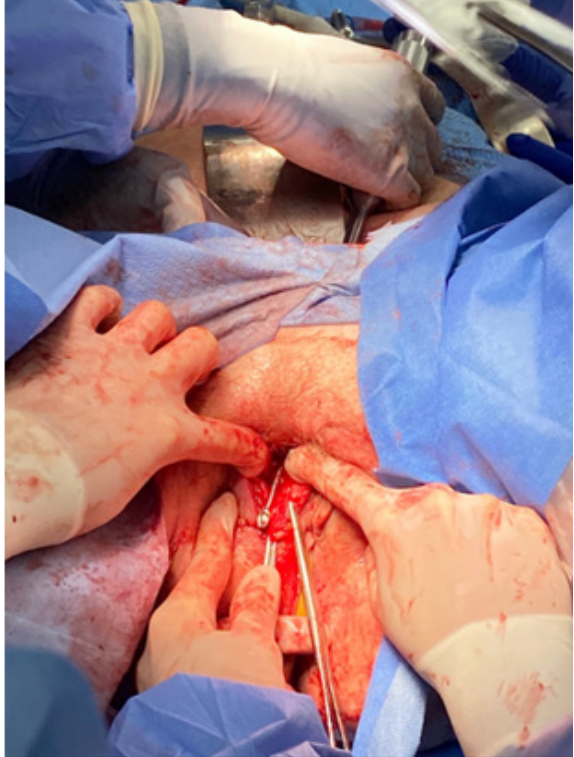

Figure 5 An abdominal approach, orthopedic team p obtained access via a Pfannenstiel incision to evaluate the pubic symphysis and surrounding bone.

On POD\# 28, during follow up evaluation in clinic, it was noted that vulvar wound was almost entirely healed (Figure 6). Five days later, she developed a diffuse, erythematous rash over her trunk and extremities. Following infectious disease recommendations, her course of antibiotics was discontinued at that time as it was suspected to be an allergic reaction. Patient was seen on POD\# 40, at which time her body rash had resolved, and her vulvar would was found to have completely healed and closed.

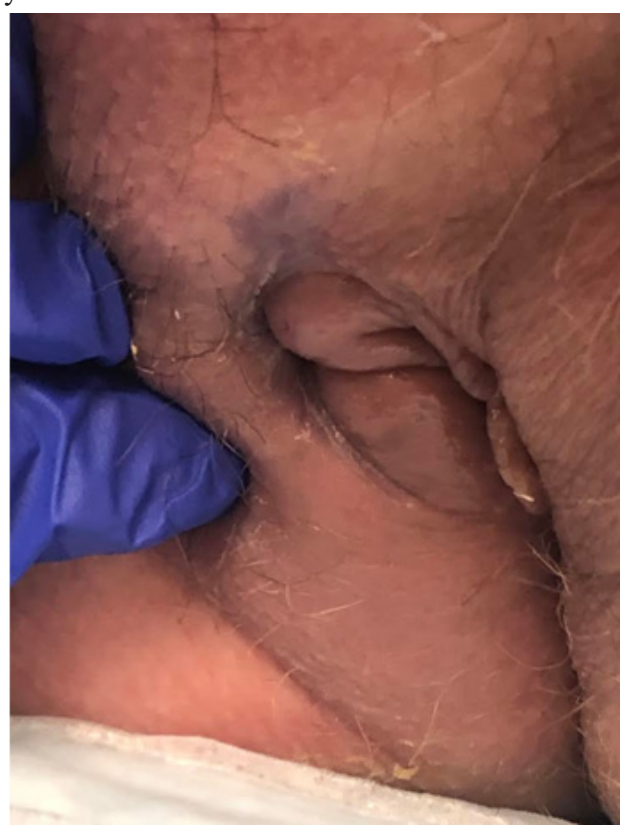

Figure 6 During follow up evaluation in clinic, it was noted that vulvar wound was almost entirely healed.

Patient presented to the clinic on POD\#47 with complaints of new onset bloody drainage from the vulvar region, and upon reevaluation was found to have a recurrence of her vulvar wound with an opening of $2 \times 3 \mathrm{~cm}$ and purulent drainage. Due to patient's complex history, she was re-admitted at that time for further multidisciplinary management. MRI of the pelvis showed persistent sinus tract from skin of right vulva to the symphysis pubis, and associated symphysis pubis osteomyelitis. Per ID recommendations, patient was initiated on start oral Linezolid 600mg twice daily. On POD\# 49 patient underwent repeat right vulvar debridement by Gynecology and pubic symphysis debridement by orthopedics. Post-operatively, daily wetto-dry wound changes and irrigation of wound were done, and patient was maintained on antibiotic therapy (Linezolid 600mg twice daily).

Patient was discharged home three days later, with daily home health nurse visits for wound care and weekly visits in Gynecology clinic. Three weeks later, patient was transitioned to daily Doxycycline $100 \mathrm{mg}$ twice daily per ID recommendations. Five weeks later, patient's vulvar wound had completely healed, with a superficial skin scar, and she was doing well clinically. She was continued on daily suppressive antibiotic therapy. On follow up evaluation two months later, patient had no evidence of vulvar wound recurrence, and she remained asymptomatic. To this day, patient has not experienced any further recurrence of her vulvar wound and pubic osteomyelitis.

\section{Discussion}

Osteomyelitis pubis is a rare infection of the inferomedial bony pelvis that causes local destruction of skeletal structures as well as adjacent soft tissue and joints such as the pubic symphysis.

Osteomyelitis can be classified into three categories based on etiology and further subdivided as acute or chronic according to duration of infection, as first proposed by Lew and Waldvogel: hematogenous osteomyelitis, contiguous focus osteomyelitis, and vascular insufficiency osteomyelitis. ${ }^{1}$ The etiology of osteomyelitis, hematogenous versus contiguous, does not appear to predict the type of microbes found.

Hematogenous osteomyelitis is considered predominantly a pediatric disease, with $85 \%$ of those diagnosed under the age of $17 .{ }^{2}$ It most often affects the metaphysis of long bones in children, particularly that of the tibia, femur, and humerus, secondary to bacteremia. The slowing of blood flow in the highly vascular metaphyseal region around the growth plate is thought to allow microbes to adhere to bone and establish infection.

More than half of osteomyelitis cases in adults are due to contiguous spread of infection to bone. Trauma and surgery are well described in literature as the leading causes, as open fractures and operative procedures such as arthroplasty can introduce pathogens via direct inoculation or surrounding soft tissue infection. Osteomyelitis pubis, specifically, is associated with gynecologic trauma including uncomplicated vaginal deliveries. ${ }^{3}$ With lengthening life expectancy amassed with advancements in surgical procedures, it stands to reason that the incidence of osteomyelitis has tripled among the elderly in a ten-year span between 2000 and 2009 in certain populations and will continue to increase. ${ }^{4}$

The third classification by Lew and Waldvogel, vascular insufficiency osteomyelitis, is arguably a description of a major risk factor rather than the source of infection. Preexisting conditions such as peripheral artery disease and diabetes that result in micro- and macrovascular compromise and neuropathy in turn lead to decubitus ulcerations, poor wound healing, and diminished supply of immune cells to clear infection. Bones of the feet are most commonly affected in such cases. ${ }^{5}$

Lew and Wadvogel's classifications of osteomyelitis are not mutually exclusive, as our patient displayed components of all three types. She had a history of diabetes, and although her only gynecologic 
history of two vaginal deliveries was remote, she developed a labial abscess potentially secondary to MRSA bacteremia which then formed a sinus tract to the pubis to allow for contiguous spread of infection to bone.

The microbe that was identified on bone biopsy of our patient's wound was Staphylococcus aureus, consistent with the most common reported findings of osteomyelitis elsewhere in the body, and comprising between $52-75 \%$ of bony infections. ${ }^{6,7}$ In a study of 81 cases of foot osteomyelitis, Senneville et al. reported a predominance of staphylococcal species in bone biopsy specimens, mainly Staphylococcus aureus and Staphylococcus epidermidis. In distant second were gram-negative bacilli such as Pseudomonas aeruginosa. Enterococci, salmonella, beta-hemolytic streptococci, viridans stretococci, escherichia coli, and enterobacter species are less common culprits. ${ }^{8}$

Osteomyelitis pubis should be considered in the differential when patients present with fever and pubic tenderness. A wide-based gait may be observed, and pain may be exacerbated by ambulation or hip abduction. Radiation to the suprapubic, perineal, inguinal, and scrotal regions may be noted.

An important distinction must be made to differentiate osteomyelitis pubis from osteitis pubis. Both conditions may present with similar signs and symptoms, however the latter is inflammatory in nature and is self-limiting while the former has an infectious etiology and may progress to catastrophic sequelae if left untreated. ${ }^{9}$

Clinical suspicion should be followed by imaging and laboratory confirmation. Bone biopsy with histopathologic examination and culture remains the gold-standard for the diagnosis of osteomyelitis, while imaging with ultrasound, plain radiograph and MRI and hematologic workup with blood cultures, complete blood count, and acute phase reactants including C-reactive protein and procalcitonin may help rule out other diagnoses with similar presentation such as osteitis pubis, puerperal endometritis, and pelvic hematoma or abscess. $^{3}$

In the presence of necrotic bone, the causative organisms can establish a biofilm which hinders the effect of antibiotics and the host's immune response, therefore antibiotic therapy alone is not sufficient for eradication of infection. Treatment of osteomyelitis begins with surgical debridement of the infected site and delay of empiric antibiotics treatment until bone biopsy is procured. When necessary, empiric therapy should include coverage of methicillinresistant Staphylococcus aureus and gram-negative organisms with IV vancomycin and third or fourth generation cephalosporins. Once the pathogen is identified on culture, antibiotic therapy should be adjusted to target the specific organism and continued for a minimum of eight weeks. In our patient, daptomycin was the antibiotic of choice due to culture proven vancomycin resistance.

\section{Summary}

Prompt diagnosis and treatment of osteomyelitis pubis which oftentimes requires prolonged periods of treatment by multidisciplinary teams yields excellent outcomes. Cosma et al. compiled a series of nine such cases in peri/post-partum women ages 25-43, all of whom achieved complete recovery after targeted antibiotic treatment with or without surgical intervention. ${ }^{3}$

\section{Acknowledgments}

None.

\section{Funding}

None.

\section{Conflicts of interest}

The author declares that there is no conflict of interest regarding this study.

\section{References}

1. Lew D, Waldvogel F. Osteomyelitis. N Engl J Med. 1997;336:999-1007.

2. Cierny G, Mader J. Adult chronic osteomyelitis. Orthopedics. 1984:15571564.

3. Cosma S, Borella F, Carosso A, et al. Osteomyelitis of the pubic symphysis caused by methicillin-resistant Staphylococcus aureus after vaginal delivery: a case report and literature review. BMC Infectious Diseases. 2019.

4. Schmitt, S. Bone and joint infections. Infectious Clinics Diseases of North America. 2017;31(2).

5. Berendt A, Peters E, Bakker K, et al. Diabetic foot osteomyelitis: a progress report on diagnosis and a systematic review of treatment. Diabetes Metab Res Rev. 2008;24(Suppl 1):S145-S161.

6. Senneville E, Melliez H, Beltrand E, et al. Culture of percutaneous bone biopsy specimens for diagnosis of diabetic foot osteomyelitis: concordance with ulcer swab cultures. Clinical Infectious Diseases. 2006;42(1):57-62.

7. Walter G, Kemmerer M, Kappler C, et al. Treatment algorithms for chronic osteomyelitis. Dtsch Arztebl Int. 2012;109:257-264.

8. Schmitt, S. Bone and joint infections. Infectious Clinics Diseases of North America. 2017;31(2).

9. Pauli S, Willemsen P, Declerck K, et al. Osteomyelitis pubis versus osteitis pubis: a case presentation and review of the literature. British Journal of Sports Medicine. 2002. 